\title{
28 Research Square \\ Specialized metabolic functions of keystone taxa sustain soil microbiome stability
}

Weibing Xun

Yunpeng Liu

Wei Li

Yi Ren

Wu Xiong

Zhihui Xu

Nan Zhang

Youzhi Miao

Qirong Shen

Ruifu Zhang

\section{Video Byte}

Keywords: soil incubation, microbial diversity and stability, co-occurrence network, machine learning, keystone function, soil, agriculture, biodiversity, Microbiome

Posted Date: February 25th, 2021

DOl: https://doi.org/10.21203/rs.3.rs-276098/v1

License: (9) (i) This work is licensed under a Creative Commons Attribution 4.0 International License. Read Full License 


\section{Abstract}

The sustainability of the terrestrial ecosystem depends on the stability of its tiniest residents. The terrestrial microbiome controls functions such as organic carbon turnover, nutrient-use efficiency, and productivity, and losing critical keystone functions may cause dramatic shifts in microbiome composition and function. A recent study sought to better understand the relationship between biodiversity and microbiome stability. Researchers inoculated microbial communities differing in phylogenetic diversity into sterilized soil and evaluated the resulting microbiome stability. They found that bacterial communities with higher phylogenetic diversity tended to be more stable throughout a range of $\mathrm{pH}$ values. Specialized metabolic functions, including "nitrogen metabolism" and "phosphonate and phosphinate metabolism," were identified as keystone functions. These critical functions were carried out by specific bacterial taxa, including Nitrospira and Gammatimonas. This study provides new insight into the relationship between soil microbiome diversity and ecosystem stability, highlighting the key role of specialized metabolic functions in maintaining a stable soil microbiome. These findings may be harnessed in the future to improve the stability of ecosystem services and guide the construction of a stable synthetic microbiota. 